\title{
Structural Health Monitoring Using Lamb Wave Reflections and Total Focusing Method for Image Reconstruction
}

\author{
Aurelia Muller ${ }^{1}$ (D) B Bradley Robertson-Welsh ${ }^{2}$. \\ Patrick Gaydecki $^{3}$ - Matthieu Gresil ${ }^{4}$. \\ Constantinos Soutis ${ }^{1}$
}

Received: 21 October 2016 / Accepted: 24 October 2016/Published online: 16 November 2016

(C) The Author(s) 2016. This article is published with open access at Springerlink.com

\begin{abstract}
This investigation aimed to adapt the total focusing method (TFM) algorithm (originated from the synthetic aperture focusing technique in digital signal processing) to accommodate a circular array of piezoelectric sensors (PZT) and characterise defects using guided wave signals for the development of a structural health monitoring system. This research presents the initial results of a broader study focusing on the development of a structural health monitoring (SHM) guided wave system for advance carbon fibre reinforced plastic (CFRP) composite materials. The current material investigated was an isotropic (aluminium) square plate with 16 transducers operating successively as emitter or sensor in pitch and catch configuration enabling the collection of 240 signals per assessment. The Lamb wave signals collected were tuned on the symmetric fundamental mode with a wavelength of $17 \mathrm{~mm}$, by setting the excitation frequency to $300 \mathrm{kHz}$. The initial condition for the imaging system, such as wave speed and transducer position, were determined with post processing of the baseline signals through a method involving the identification of the waves reflected from the free edge of the plate. The imaging algorithm was adapted to accommodate multiple
\end{abstract}

Aurelia Muller

aurelia.muller@postgrad.manchester.ac.uk

Bradley Robertson-Welsh

bradley.robertsonwelsh@manchester.ac.uk

1 Aerospace Research Institute, University of Manchester, Sackville Street, Manchester M139PL, UK

2 School of Mechanical, Aerospace and Civil Engineering, University of Manchester, Sackville Street, Manchester M139PL, UK

3 School of Electrical and Electronic Engineering, University of Manchester, Sackville Street, Manchester M139PL, UK

4 i-Composites lab, School of Materials, University of Manchester, Sackville Street, Manchester M139PL, UK 
transmitting transducers in random positions. A circular defect of $10 \mathrm{~mm}$ in diameter was drilled in the plate, which is similar to the delamination size introduced by a low velocity impact event in a composite plate. Images were obtained by applying the TFM to the baseline signals, Test 1 data (corresponding to the signals obtained after introduction of the defect) and to the data derived from the subtraction of the baseline to the Test 1 signals. The result shows that despite the damage diameter being $40 \%$ smaller than the wavelength, the image (of the subtracted baseline data) demonstrated that the system can locate where the waves were reflected from the defect boundary. In other words, the contour of the damaged area was highlighted enabling its size and position to be determined.

Keywords Lamb waves - Structural Health Monitoring · Defect imaging - Image reconstruction · Total Focusing Method · Lamb waves reflections · Signal processing · Full Matrix Capture · Symmetric fundamental mode $\cdot$ Circular damage

\section{Introduction}

An increasing amount of research is focusing on the development of structural health monitoring systems either for the development of smart or self-sensing composite structures, or to increase the operating life of ageing metallic structures. Several studies have demonstrated the ability of guided waves to be used as a damage sensing method in composite materials and metals. When combined with a network of piezoelectric disk transducers, known as PZT or Piezoelectric Wafer Active Sensor, they represent a relatively non-invasive structural health monitoring system whilst providing a relatively large area of investigation (generally up to few meters depending on the material damping properties). The waves can be generated and sensed with PZT, with the output in the form of a signal or A-scan (amplitude against time graph) varying with the propagation medium. Guided waves sensing is based on: (1) the detection of changes in the signal waveform compared to previous signals or a baseline signal (representative of the material in its pristine condition); (2) the interpretation of those changes into information about materials and any defects (such as quantity, size and location). In addition, regular assessment can be used to estimate the growth rate of a defects, and the remaining time before repair or replacement must be carried out on the structure. However, the interpretation becomes more complex and time consuming as the number of signals increases, ultimately it is inefficient when analysing multiple data from an array of sensors. The use of digital signal processing techniques can provide automated interpretation of a large amount of signals. In particular, imaging techniques such as the total focusing method (TFM) with full matrix capture (FMC) has been previously used to process signals obtained from multiple transmitting or receiving transducers in a linear array.

The aim of this study is to develop a defect imaging system for structural health monitoring based on Lamb waves and the TFM that can analyse data from multiple transmitter and receiver PZT transducers in a circular array. A TFM algorithm with FMC was adapted to a circular array of PZT transducers, each of them acting successively as transmitter or receiver. The initial condition of the imaging system, such as wave speed and sensor position, was determined with a method based on determining the relative position of the plate boundaries, using the wave edge reflections. The imaging system was experimentally tested by creating a damaged area as a circular defect. Once confidence has been built, the technique will be applied to composite plates where the changes in wave velocity and damping with respect to the fibre orientation will be taken into account in the algorithm. 


\section{Structural Inspection Using Lamb Waves}

\subsection{Principle of Lamb Wave Testing With Piezoelectric Transducers}

Lamb waves, also known as guided waves, can be defined as a type of ultrasonic elastic wave propagating in a solid medium and guided between two parallel free surfaces, such as the upper and lower surface of a thin plate [1,2]. Their propagation is dispersive, meaning that the wave's velocity varies with the plate thickness and the frequency at which they are generated, and that different modes (groups of waves with different particle motion and velocities) can exist simultaneously. In theory, an infinite number of wave modes exist at different excitation frequencies, each of them having different wavelengths and velocities. These modes are generally classified in two types, symmetric or antisymmetric, depending on the particle motion their propagation generates. The symmetric modes (designated S0, S1, \{..., $\mathrm{Sn}$ ) have an in-plane particle motion, parallel to the wave propagation direction (associated to axial plate or compression waves). The antisymmetric modes (designated A0, A1, \{... An) have a particle motion perpendicular (or out-of-plane) to the propagation direction of the wave [2]. Both mode types propagate through the whole of the plate thickness and over relatively long distances, depending on the damping of the propagation medium, this can be in the range of dozens of centimetres to a few meters, making Lamb waves suitable for structural health assessment.

In SHM applications, piezoelectric (PZT) disc transducers (also known as piezoelectric wafer active sensors) are often used in conjunction with Lamb waves. They can be permanently implemented on the material to monitor and are relatively unobtrusive (often around $0.5 \mathrm{~mm}$ thick and a few millimetres wide). In addition to this, a major advantage of PZT is their ability to both generate and sense Lamb waves, enabling their use in various networks and configurations, such as pitch and catch (waves emitted and received by different PZT) or pulse echo (waves emitted and received with the same PZT) [3].

\subsection{Lamb Wave Interactions with Damage and Boundaries}

Damage sensing with Lamb waves is based on the observation of alteration in the signal waveform due to changes in the propagation medium. Lamb wave signals are obtained in the form of A-scans (graphs of the amplitude evolution over time). The first signals collected are used as a baseline, meaning that they are assumed to be representative of an undamaged material. The potential defects are then detected by comparing the baseline with the subsequent collected signals [4]. One limitation of this technique, however, is that this prevents the detection of inherent defects. In addition to this, several investigations observed that the wave propagation is often only altered when the signal wavelength is smaller than the defect sizes [2, $5,6]$. Some of the common changes observed in the signal waveform include: delay in the arrival time or time of flight (ToF) of a mode [5], presence of new reflections, local attenuation [6], or mode conversion phenomenon [7, 8]. Mode conversion is related to the transformation of one mode into another which can exist under the same conditions. For instance, Willberg et al. [6] reported the conversion of an S0 mode into an A0 mode after its interaction with a hole in a CFRP composite. This phenomenon can also occur when waves are reflected from the boundaries of the material under investigation [9]. Shantanam and Demirli [9] investigated the interaction of an obliquely incident wave of different frequencies with the free edge of an aluminum plate. They found that $\mathrm{A} 0$ and $\mathrm{S} 0$ modes produced multiple mode reflections. For 
instance, the reflection of an incident S0 was split between S0 and SH0 (shear waves), while A0 was split between A0, A1 and SH1. However, the energy of the incident mode was not divided equally between the reflected modes. When the A0 mode was incident, the reflected A0-mode often had a lower energy than the other created modes, and was therefore difficult to detect. In contrast, when the S0 mode was incident, it consistently exhibited a detectable reflected S0 mode. Reflections were specular when the reflected wave consisted of the same mode as the incident wave.

\section{Principle and Evolution of Defect Imaging With Total Focusing Method}

Recent studies focused on the development of guided wave imaging systems in the time domain, including, through the use of triangulation [10], sum and delay beam forming [11-14] or tomographic reconstruction [15]. Triangulation requires a limited number of sensors and provides a limited resolution [10]. Similarly, the resolution of the tomographic reconstruction improves when increasing the number of sensors used, however it is often limited to investigations within the array of sensors [15]. Sum and delay beam forming generally provides an improved resolution for a limited number of sensors, and is less sensitive to noise compared to other imaging techniques making it suitable for structural health monitoring applications [12]. However, sum and delay beam forming can refer to different processing techniques and equations, including the Synthetic Aperture Focusing technique (SAFT) and the total focusing method (TFM) [12-16]. Ultimately, most of the sum and delay beam forming techniques originate from SAFT.

SAFT was initially developed for airborne radar mapping systems in the 1950s [17-19]. In the field of Digital Signal Processing (DSP) it refers to a process in which a large aperture (probe width) focused transducer is simulated, or synthesised using a series of measurements obtained with one, or several, small aperture transducers moving over a surface $[17,20,21]$. In other words, a uniform resolution is achieved by combining signals taken from multiple low resolution transducers located on the surface of the test piece. The signal waveforms are combined through a process of summation and time shifting that takes into account spatial consideration, such as wave velocities and propagation paths across the surface or though the volume [22]. The signal obtained from the sensor the closest to the emitting transducer corresponds to the shortest travelled time and often become the reference signal; the other signals are shifted according to it. Accurate knowledge of those characteristics, and particularly the wave speed and probe position, are essential for a successful and precise application of SAFT. Through this process SAFT leads to an enhanced signal to noise ratio (SNR), and resolution while avoiding the trade-off between the resolution and range of inspection which arises when selecting a single transducer.

Around the 1980s, its application was extended to non-destructive ultrasonic testing of materials to improve detection and location as well as the quantification of defects. Extensive literature describes the uses of SAFT to investigate concrete structures [23-26] and metals, including aluminium [19-21] and steel pipes [14, 27]. Although this type of ultrasonic imaging has been widely used for Non-destructive Evaluation (NDE) with a moving array of sensors, its application to structural health monitoring is not straightforward and requires the algorithm to be adapted to an array of multiple static transducers. In the last decade, several researchers modified SAFT in order to use it for the health monitoring of aluminium and composite structures with PZT and guided waves. Also, the algorithm was adapted for different 
configurations such as pulse-echo [11] and later pitch and catch [28]. Finally, the SAFT evolved into the TFM. This algorithm creates an image by combining the signals obtained from multiple transmitters and receivers, this combination is referred as full matrix capture (FMC) $[12,14,16]$. The main challenge of TFM with FMC is that, contrary to other SAFT or sum and delay beam forming methods, there is no unique referenced signal. Instead, the reference changes with the successive variation of coordinates of the transmitter transducer.

A particular issue of TFM with FMC lies in multiple reflections of guided waves from the plate boundaries. They generate artefacts making it difficult to differentiate between pixels representing artefacts and actual defects [13]. A solution is to enhance changes due to damage by subtracting the baseline signal to the test signal after each assessment [10]. However, this method can degrade the system's ability to distinguish defects inherent to the structure.

\section{Experimental Setup and Data Collection}

\subsection{Material, Sensor and Instrumentation}

The material under investigation was an AW 1050A-H14 aluminium (supplied by Smith Metal), square plate of dimensions: $1 \mathrm{~m} \times 1 \mathrm{~m} \times 2 \mathrm{~mm}$. A set of 16 piezoelectric disc transducers (PZT), also known as piezoelectric wafer active sensors (PWAS), was bonded to the plate surface. A schematic of the plate with the sensor locations is shown in Fig. 1. The main characteristics of the PZT (supplied by PI ceramics) are listed as follows: the diameter is $10 \mathrm{~mm}$, the thickness is $0.5 \mathrm{~mm}$, the resonance frequency is $200 \mathrm{kHz}$, the frequency coefficient is $2000 \mathrm{~Hz} \cdot \mathrm{m}^{-1}$, and the mechanical quality factor is 80 (which is a manufacturer specification).

The experimental instrumention is comprised of a Tektronix AFG3052C function generator and a DPO5034B Tektronix oscilloscpe, with the setup for data collection shown in Fig. 2. The transmission and reception of lamb wave signals with the PZT was performed in pitch and catch configuration. Each transducer acted either as an emitter or a receiver, enabling the collection of 240 signals in total. The generator sent a three cycle tone burst excitation signal of $10 \mathrm{~V}$ maxmimum amplitude to the transmitting PZT and the oscilloscope. The excitation frequency was selected based on the analysis of the dispersion and tuning curve.

\subsection{Experimental Procedure}

The dispersion and tuning curve enables the observation of the amplitude and velocity variation of the different modes as a function of the excitation frequency. In this investigation, the experimental dispersion and tuning curves, such as shown in Figs. 3 and 4, were obtained by varying the excitation frequency of the transmitted signal between $30 \mathrm{kHz}$ and $600 \mathrm{kHz}$. They were then compared with theoretical data (obtained from 'WaveFormRevealer 1.0' LAMSS (C) 2011) [29].

Based on the tuning and dispersion curves, the excitation frequency selected for the remaining experiment was $300 \mathrm{kHz}$. At this frequency the fundamental symmetric mode (S0) amplitude reaches a maximum, whilst the fundamental antisymmetric mode (A0) is highly attenuated (such as shown in Fig. 4). Therefore, the dispersion effect of lamb waves is reduced. According to the dispersion curve in Fig. 4, the experimental group velocity of the $\mathrm{S} 0$ mode is in the region of $5000 \mathrm{~m} . \mathrm{s}^{-1}$ leading to a wavelength of approximately $17 \mathrm{~mm}$. This is similar to that measured for a quasi-isotropic CFRP laminate [30]. 


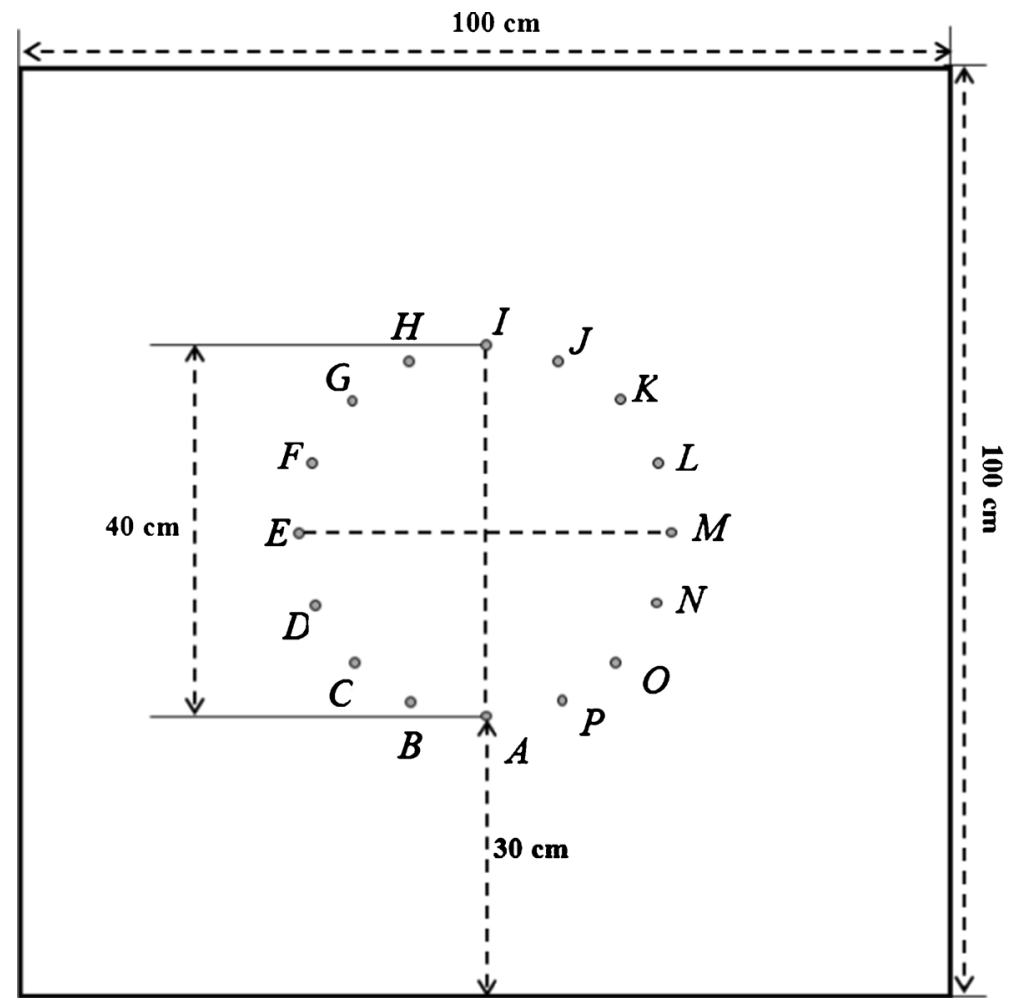

Fig. 1 Schematic of the aluminium plate dimensions and the position of the 16 bonded piezoelectric sensors (named from A to P)

After selection of the excitation frequency, three sets of data were collected with each of them containing 240 signals. The first data collected were the baseline signals of the plate in an undamaged state. The second set of data, called Test 1 , corresponds to the signal collected after

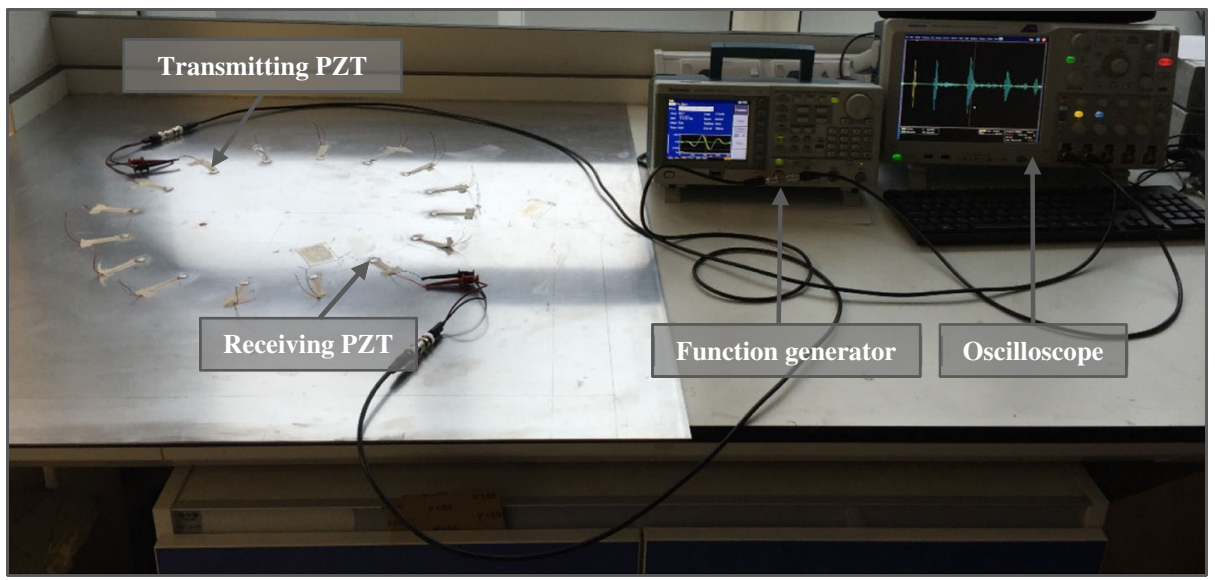

Fig. 2 Picture of the instrumentation: the generator transfers a 3 tone burst excitation signal to the oscilloscope, and on to a PZT emitter that generates guided waves in the plate. A PZT sensor then receives the wave's signal, and the voltage is transferred to the oscilloscope 


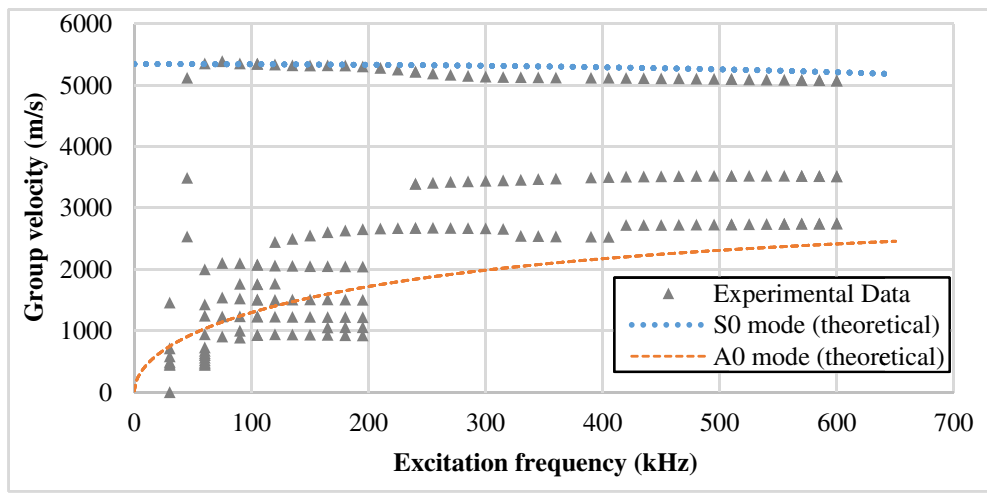

Fig. 3 Dispersion curve of the $2 \mathrm{~mm}$ thick aluminium plate

the plate was damaged. A circular damage (D1) of $10 \mathrm{~mm}$ diameter was drilled into the plate; its location is represented in Fig. 5. Finally a third set of data, called Test 2, was collected and additionally included two simulated defects SD1 and SD2, such as shown in Fig. 5. These defects were simulated by coupling two metal blocks to the metallic plate with ultrasonic gel. The area covered by SD1 was $59 \mathrm{~mm} \times 38 \mathrm{~mm}$ (with a metal block of $759 \mathrm{~g}$ ), while SD2 covered an area of $54 \mathrm{~mm} \times 37 \mathrm{~mm}$ (with a metal block of $730 \mathrm{~g}$ ).

\section{Post Processing of the Baseline Signal}

\subsection{Determining the Relative Transducer Positions}

The method for post-processing the baseline signal was first presented at the 8th European Workshop on Structural Health Monitoring (EWSHM2016). Figure 6 shows an example normalised amplitude against time graph (A-scan), with the transmitted signal and received signal. Given that the excitation frequency of the signal $(300 \mathrm{kHz})$ is tuned on the $\mathrm{S} 0$ mode, the A0 mode, whilst present, is heavily attenuated. The consequence of this is that the S0 excitations are clearly observable. The first response corresponds to the direct path between the transmitter and receiver with the second response produced by a boundary reflection.
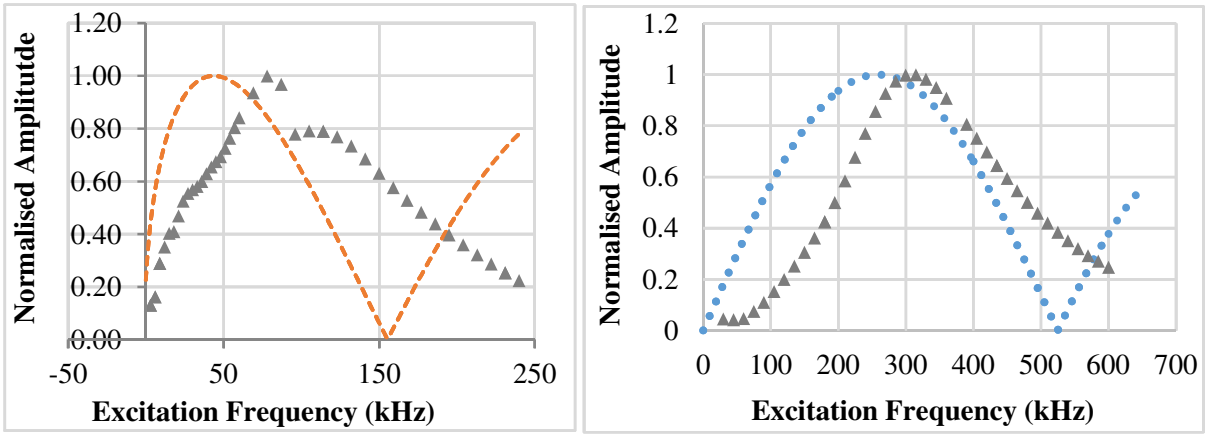

Fig. 4 Tuning curves of the antisymmetric fundamental mode (left) and the symmetric fundamental mode (right) the le gend colour code is shown in Fig. 3 


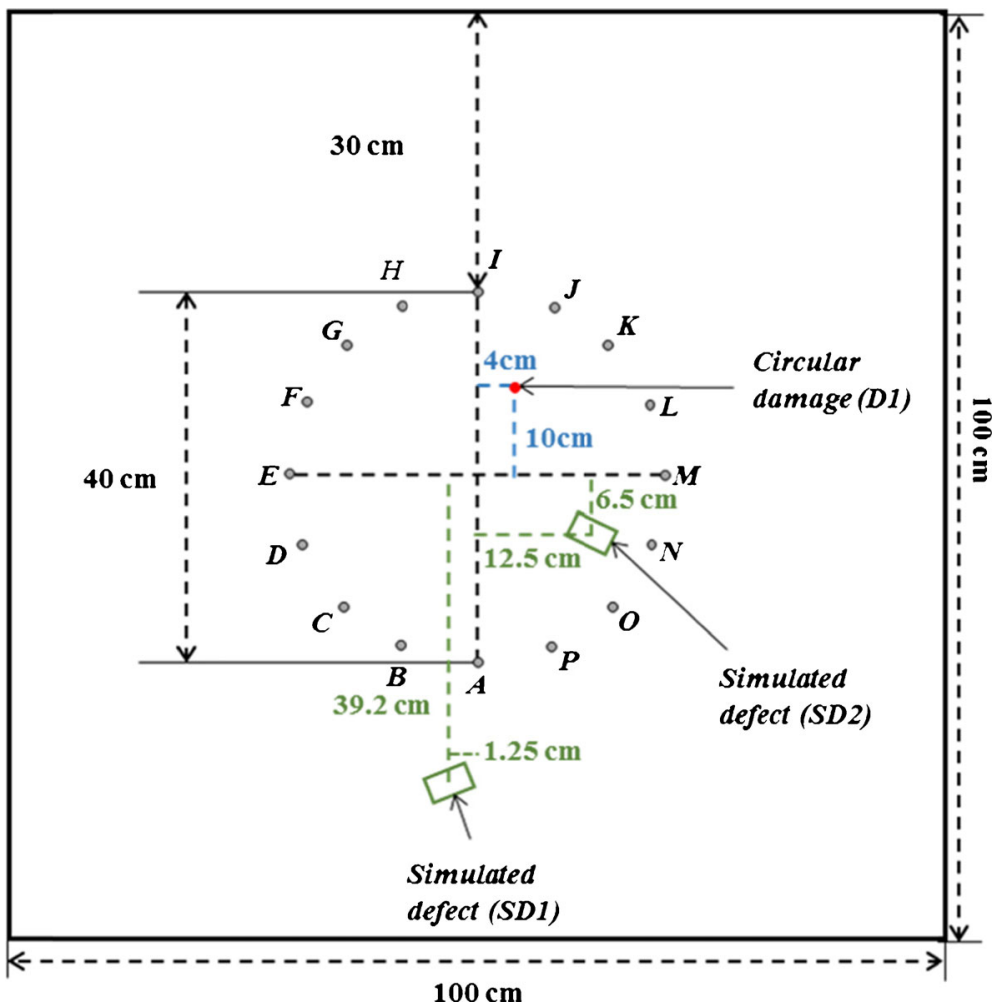

Fig. 5 Schematic of the location of the circular damage related to Test 1, and the two simulated defects in the form of a coupling defect related to Test 2

Taking a Hilbert transform of the transmitted and received signals, the time of the first peak of the received signal can be subtracted from the time of the peak of the transmitted signal. This, subtracted value, corresponds to the direct path time of flight from the transmitter to the sensor. Calculating the time of flight for all of the transmitter/sensor combinations, and then applying a triangulation algorithm allows for the relative position of all of the transducers in the time domain to be experimentally obtained.

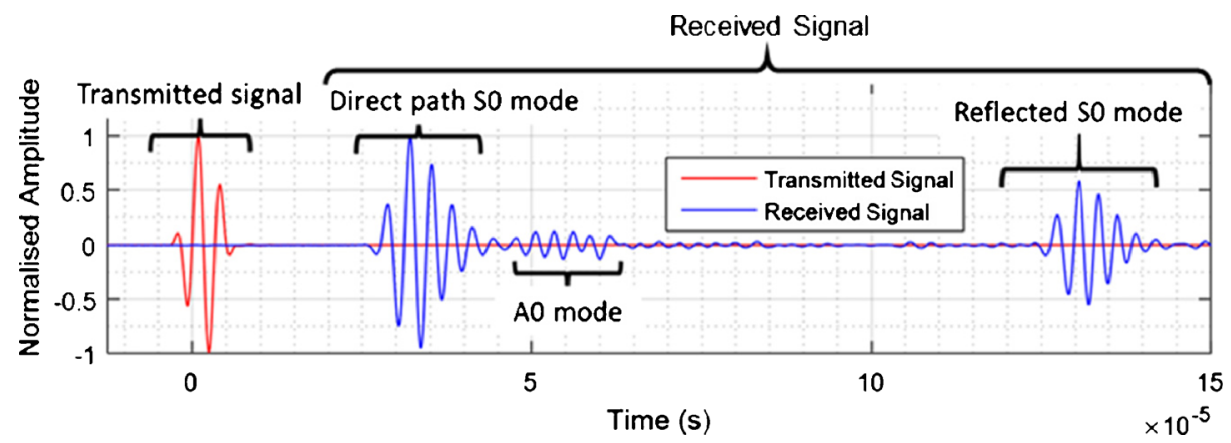

Fig. 6 Example A-scan showing the normalised transmitted and received signals, and their related modes 


\subsection{Error Analysis of Equivalent Signals}

So as to identify any systematic experimental errors, and quantify the post-processing error, the time of flight from one transmitter to another sensor was compared with the time of flight of the return path. Equation 1 shows an example error calculation for the case where 'A' and ' $\mathrm{B}$ ' are the two transducers.

$$
\text { Error }=100 \times\left(\frac{t_{A B}-t_{B A}}{0.5 \times\left(t_{A B}+t_{B A}\right)}\right)
$$

Figure 7 shows the percentage error for each transducer. It is assumed that the error presented is within acceptable limits (maximum error is less than $0.5 \%$ ) and randomly distributed: consequently, there is assumed to be no systematic error present, and both the experimental measurements, Hilbert transform, and time of flight calculations are considered valid.

\subsection{Determining the Location and Orientation of Plate Edges}

Consider the time of flight of the second, reflected, wave packet (labelled in Fig. 6 as the reflected S0 mode). Previous literature [9] has indicated that when the S0 mode hits the free edge of a plate, the wave reflection is specular, that is to say, the wave is reflected at the same angle and at the same speed as the incident wave. If it is further assumed that only a single boundary reflection occurs, then the relative position and orientation of the plate edges can be calculated. Knowing the time of flight of both the direct, and reflected path, the solution defining the locations and orientations of all possible edges is described by an ellipse. The two foci of the ellipse are the sensor and transmitter locations, with the ellipse itself defining all possible edge locations. The tangent to the ellipse at each point defines the necessary orientation of the edge in order for the reflection to be specular at that point, which is illustrated in Fig. 8.

Considering two sensor/transmitter pairs, the potential edge reflections are described as two ellipses. Two ellipses have a maximum of two tangents in common, consequently, applying this technique to multiple sensor/transmitter pairs and considering only the common ellipse tangents as potential edges quickly narrows down the possible locations for edges. Note that it is not expected that all reflections will be specular, however, common tangents which occur only between two

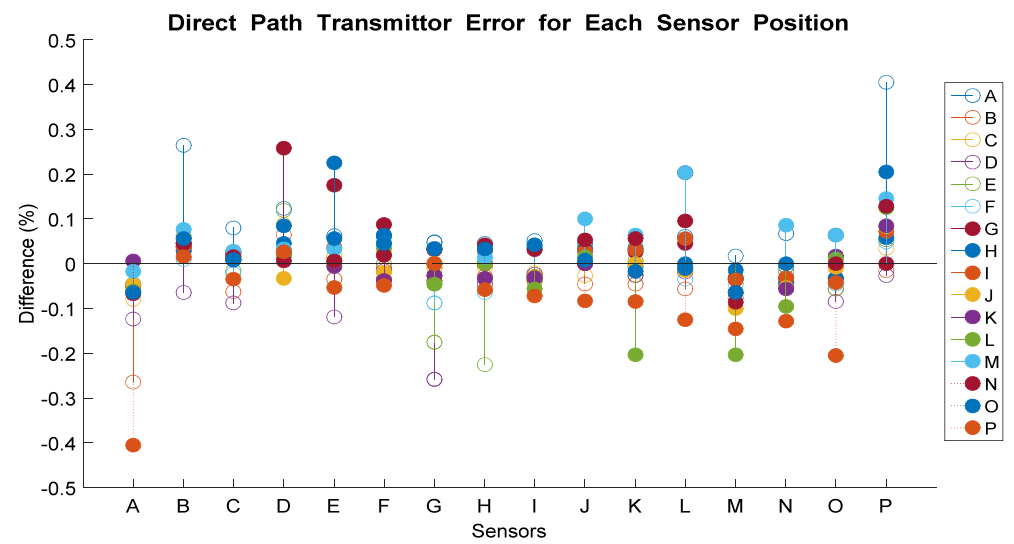

Fig. 7 Distribution of percentage difference of the direct path time of flight from one transducer to another, c ompared with it's return path. Note that this chart is shown relative to each sensor position 

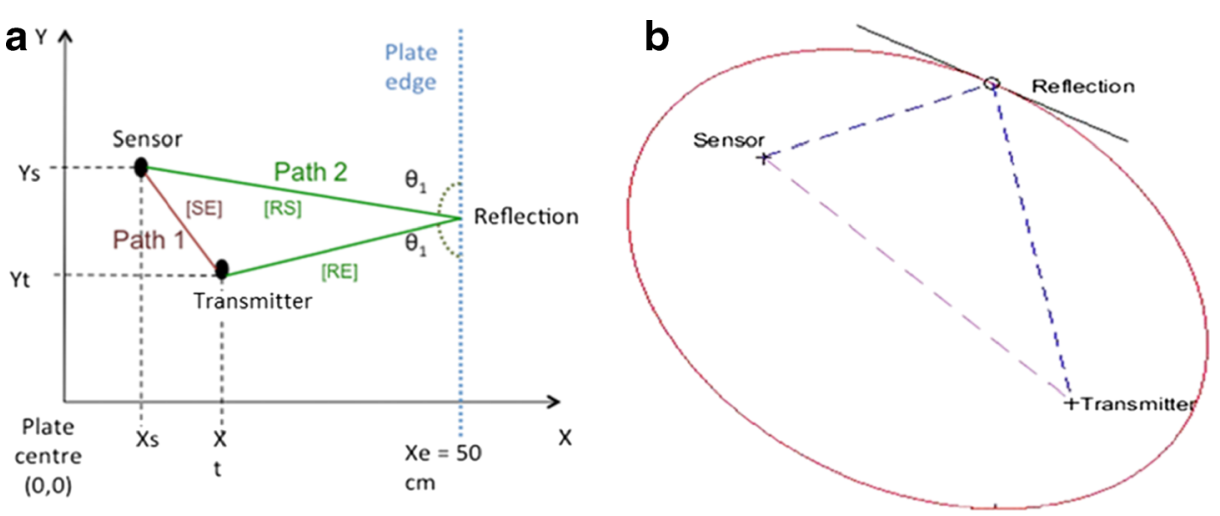

Fig. 8 (a) shows an assumed specular reflection from a plate boundary. Path 1 and Path 2 correspond to the distance tr avelled on the direct path, and reflected path, respectively. (b) shows a more conceptual view, with all potential reflection points described by an ellipse, obtained from the known distances of Path 1 and Path 2

ellipses (defined herein as unique solutions) are discarded, and common tangents occurring for three or more ellipses are assumed as edges. Figure 9 shows all of the ellipses plotted in the time domain when the transducer, 'A', is transmitting (all transducers are in their triangulated positions relative to 'A'). In Fig. 9, common tangents are indicated as dotted black lines between red dots, with the common tangent representing multiple (ie more than three) ellipses shown as a blue line.

From Section 5.2, the maximum error in the time of flight calculations between a transmitter and sensor, and the return path, is $0.5 \%$. Given that two sensor/transmitter pairs are required in order to form a common tangent, a common tangent is defined as the case where the magnitude of the difference between both the y-intercept and gradient of the two-sensor/ transmitter pairs is less than $1 \%$.

\subsection{Determination of Wave Speed and Transition to Spatial Domain}

Determining the possible edge locations for all transmitter/sensor pairs, four edges become apparent. Applying the knowledge that the plate is square, and of dimensions $1 \mathrm{~m} \mathrm{x} 1 \mathrm{~m}$, allows a transition from the time domain to the spatial domain. This is shown in Fig. 10, where the transducer positions calculated via triangulation (blue), are plotted alongside the assumed sensor position (red).

In converting to the spatial domain, the wave speed can also be determined by substituting the plate dimensions in both the time and spatial domain into the wave speed equation (Eq. 2):

$$
c_{S 0}=\frac{\text { distance }}{\text { time }}=\frac{1}{0.00019527}=5121.1{\mathrm{~m} . \mathrm{s}^{-1}}^{-1}
$$

The group velocity $\left(5121.1 \mathrm{~ms}^{-1}\right.$ ) corresponds well to the wave speed calculated in Section 4.2 from the tuning curves (Fig. 3). Consequently, the method to determine the plate boundaries is validated, and the specific paths which have produced a tangent common with the verified edges can be considered accurate. These wave paths are shown as red, alongside the direct paths in black, in Fig. 11.

To summarise, the post-processing of the baseline signal achieves the following:

1) Transducers are positioned in the time domain via triangulation of the direct path time of flight. 


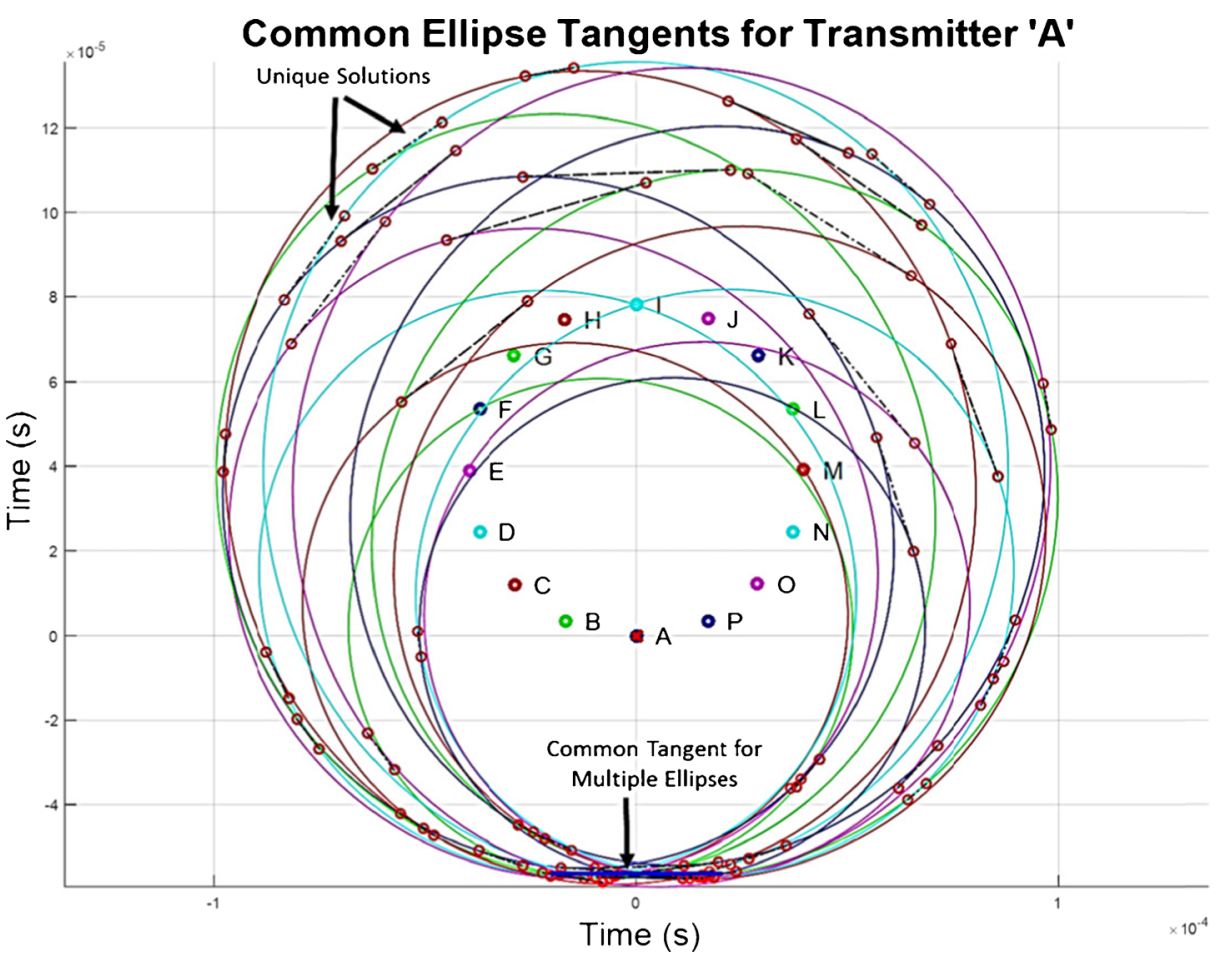

Fig. 9 All ellipses constructed from sensors paired with transmitter 'A'. Note that common tangents ar e illustrated as dashed lines between (black) around dots (red), only one tangent in this case is considered to be shared by more than two ellipses (shown by the arrow and bleu line)

2) The direct path time of flight between two transducer pairs is compared with its return time of flight, the subsequent error is quantified as less than $0.5 \%$ for all transducer pairs.

3) The second wave packet, assumed to be a specular boundary reflection, is used to calculate the potential location and orientation of the plate boundaries in the time domain,
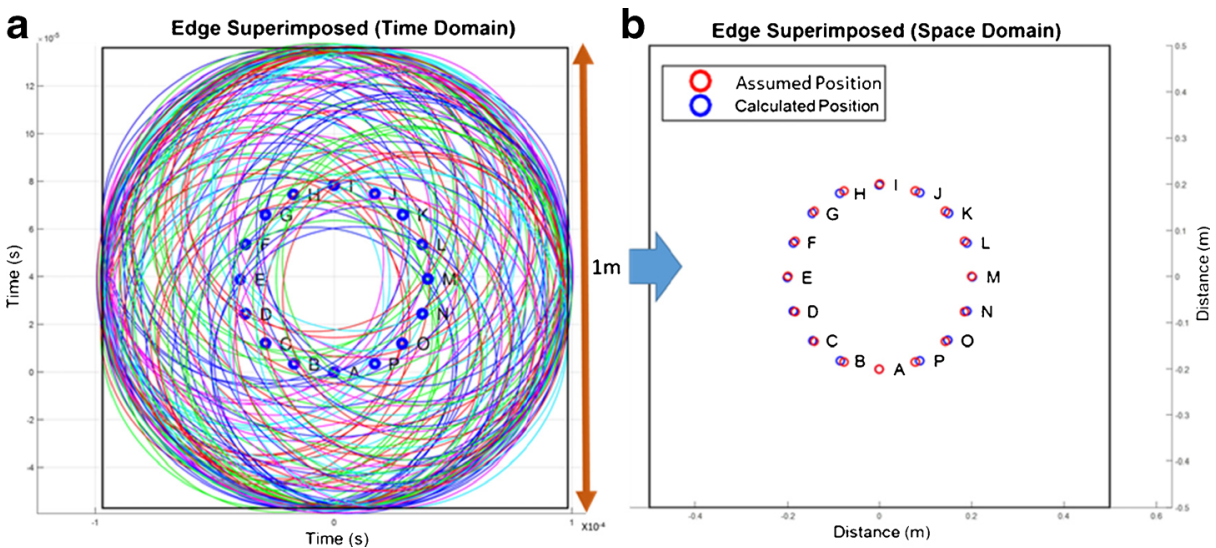

Fig. 10 Ellipses, corresponding from all transducer pair combinations provide four clear edges, which represent the plates' maximum dimensions. Insertting the physically measured dimensions allows the transition from the time domain (a) to the spatial domain (b), where the triangulated positions of the transducers (blue) are shown alongside the assumed positions (red) 


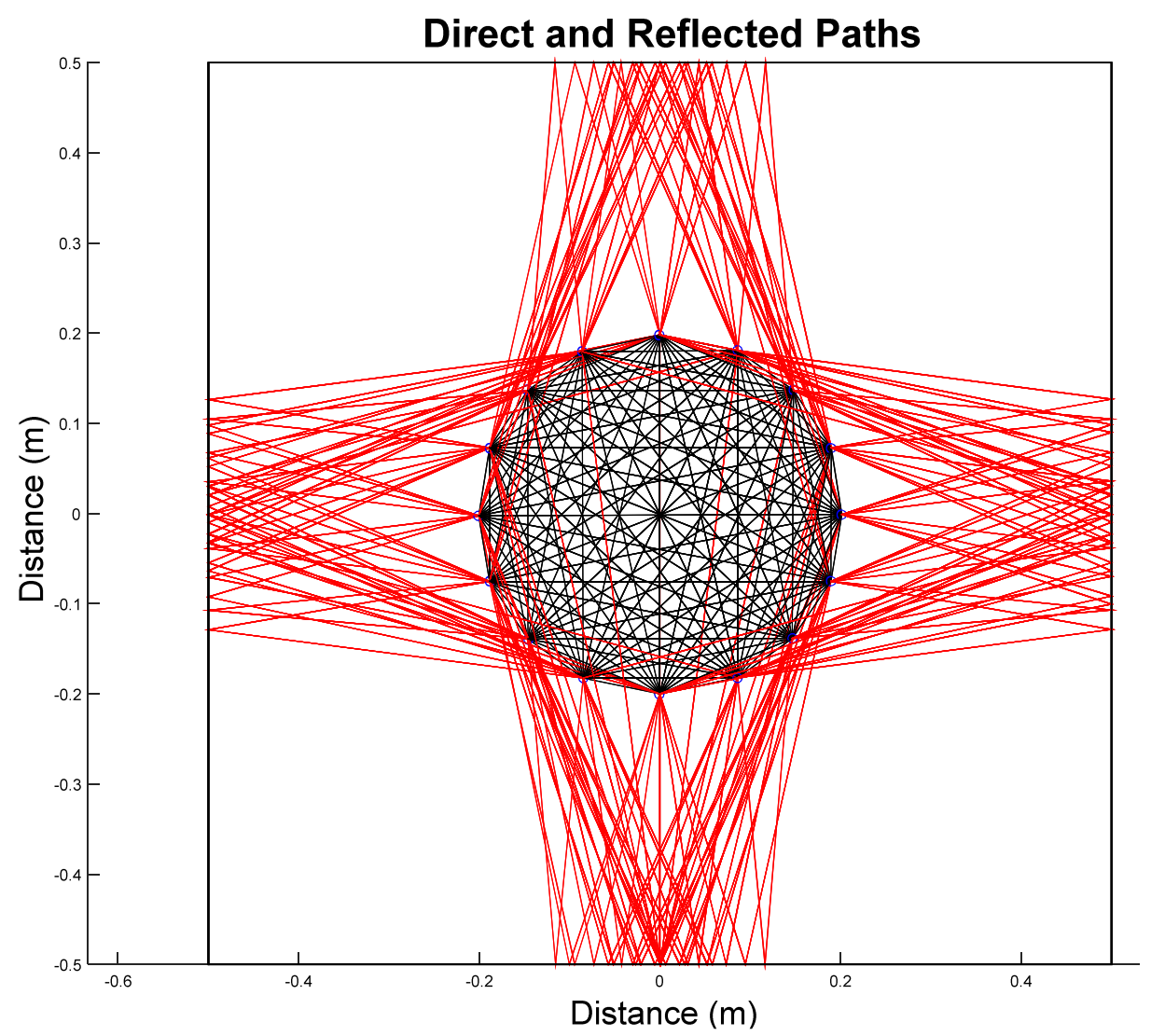

Fig. 11 Diagram showing direct propagation paths (black), and reflected propagation paths (red). Note that only the reflected paths which share common tangents (ie demonstrate specular reflection) are shown

through comparing ellipse tangents (constructed with the transducer pair as foci). Where three or more ellipse tangents are common, a boundary is assumed.

4) Applying this method to all transducer pairs shows four clear edges. Applying the knowledge that the plate is a square, and inputting the maximum dimension of the plate allows the transition to the spatial domain, and the calculation of the wave speed.

5) In comparing the wave speed against what is expected from the tuning curves, specular reflected wave paths are validated, extending the potential region of inspection.

\section{Resulting Signal for Simulated and Actual Damage}

\subsection{Circular Damage (Test 1)}

The A-scans shown in Fig. 12 are for all sensors when transducer ' $\mathrm{I}$ ' is transmitting. The black circles represent the point in time at which a specular reflection is expected as a consequence of the circular hole (refer to D1 in Fig. 5). Attenuation is seen on the signal I-N (highlighted in the top right corner of Fig. 12). The path I-N passes close to the hole, but it is still surprising that even a small amount of attenuation is present, given that the signal wavelength of $17 \mathrm{~mm}$ 


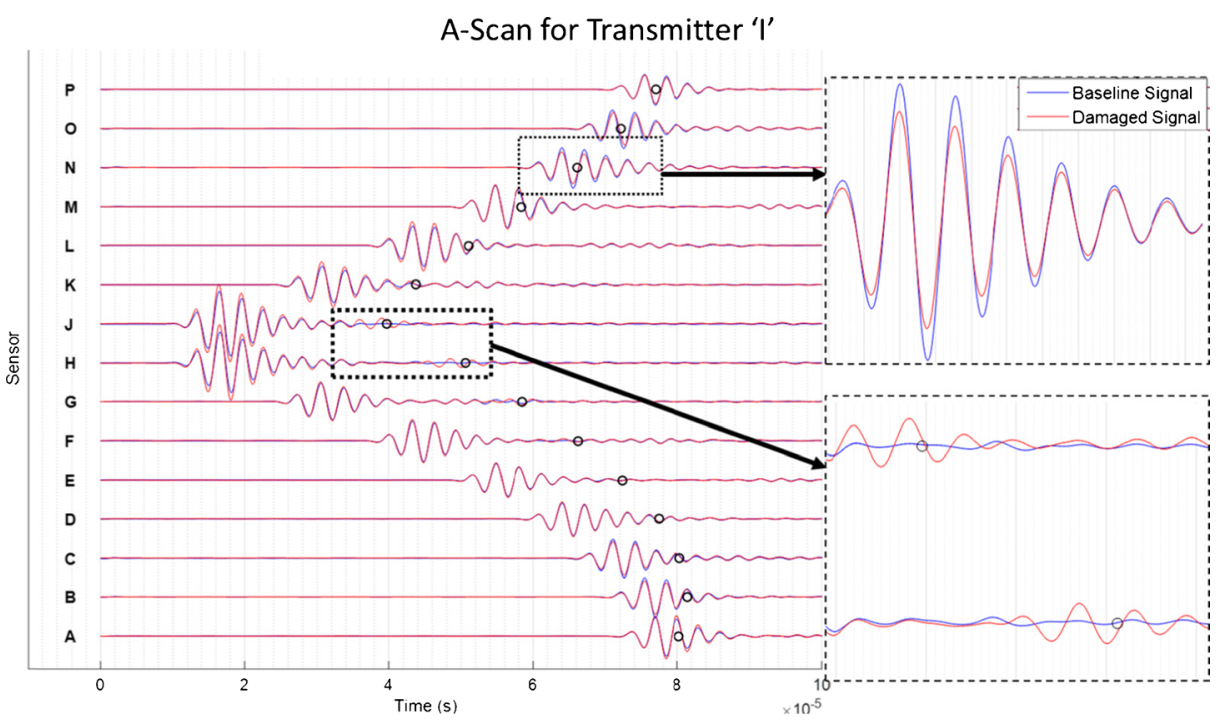

Fig. 12 A-Scan of baseline and damaged signals for each sensor when 'I' is acting as transmitter. The black circles repre sent where damage is expected to occur due to the presence of specular reflections from the circular damage

(calculated from the wave speed) is smaller than the $10 \mathrm{~mm}$ diameter of the hole. On the signal I-J and I-H, a small increase in amplitude is witnessed over the black circles (highlighted in the lower right corner of Fig. 12). This suggests that there is an additional, specular reflection is present as a consequence of the circular damage.

\subsection{Signal Obtained With Coupling Defect (Test 2)}

The A-scans shown in Fig. 13 are for all sensors when transducer ' $A$ ' is transmitting. The signals A-N, A-M and A-L all show attenuation on the first wave packet, relating to the direct

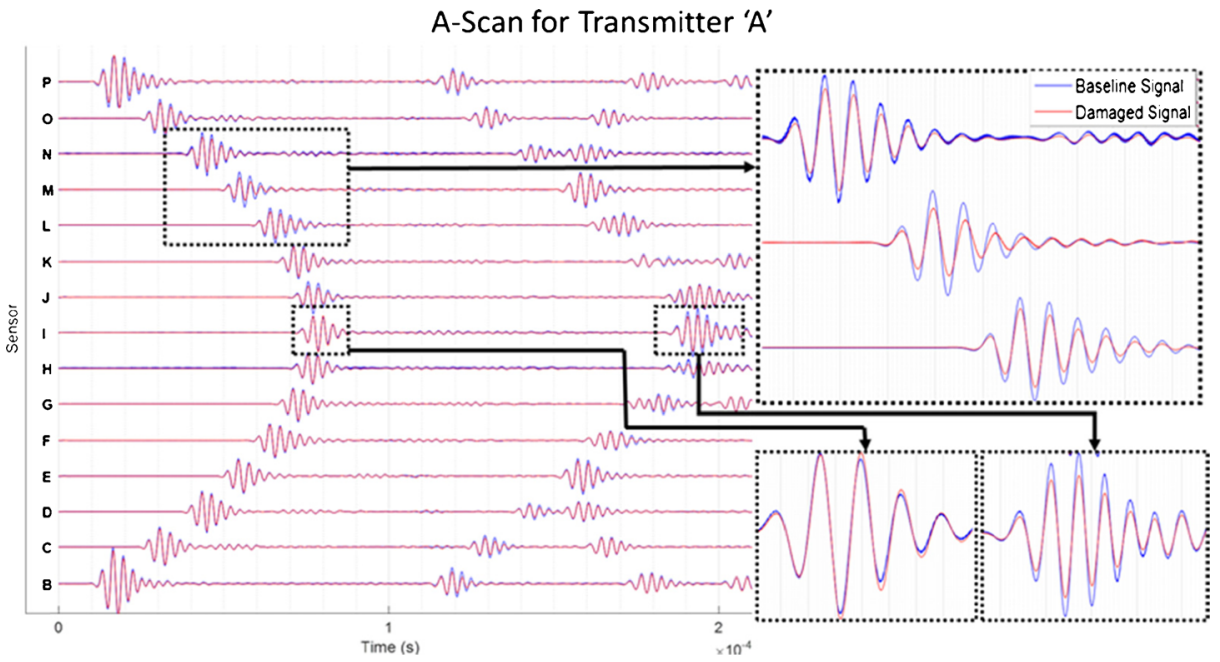

Fig. 13 A-Scan of baseline and damaged signals for each sensor when 'A' is acting as transmitter 
path (highlighted in the upper right corner of Fig. 13). Given that the damage, SD2 (Fig. 5) lies on those paths, it is reasonable to assume that the coupling defect afforded by SD2 is responsible for this attenuation. Interestingly, A-I shows no attenuation on the first wave packet, but the second wave packet, indicating the reflected path, does show attenuation (lower right of Fig. 13). SD1 (Fig. 5), lies on the reflected path (Fig. 11), and it can be concluded that this coupling defect is being detected here.

\section{Lamb Waves Imaging}

\subsection{Design of the TFM Algorithm}

As described in the literature review, the total focusing method (TFM) is a sum and delay beamforming imaging system involving multiple transducers at different locations. It aligns the time axis of different signals to focus them on each pixel. In other words, the time (defined by the transmitter and receiver location) taken by each signal to reach a specific pixel are calculated and compared. The signal with the minimum travelled distance often becomes the reference. The delay calculated between the reference time and the other travel times (from other signals) is subtracted to the time axis of its respective signal. Then, the amplitudes related to that pixel are extracted from each signal and added. Finally the total value is assigned as the pixel intensity. It must be noted that the envelope of the signal is often used instead of the signal itself. However, the complexity of this system increases when considering multiple transmitting transducers as it creates multiple references. The full matrix capture (FMC) implies that the position of a transmitting transducer can successively become the position of a receiving transducer and vice versa. In this process a pixel matrix (or grid) is calculated for each transmitting transducer following the TFM, then all the matrices are added forming a sum total matrix. Through this process the FMC algorithm enables the maximum amount of information to be collected, by making use of the PZT's ability to both transmit and sense Lamb waves. Previous investigations used TFM with FMC for different non-destructive testing systems using linear array of sensors [16].

In this investigation, Eq. 3 accommodates the random positioning of the transducers. This was achieved by calculating the distance travelled between each pair of transducers and a pixel in Cartesian coordinates. The travelled distance is then converted into travelled time by introducing the wave speed previously calculated. The amplitude in the signal's envelope (relative to the transducer pair) associated to this travelled time is extracted and added to the pixel intensity. This operation is repeated for each transmitter/receiver pair.

$$
I_{(x, y)}=\left|\sum_{i=1}^{N} \sum_{j=1}^{N} h_{T_{i}, R_{j}}\left(\frac{\sqrt{\left(x_{T_{i}}-x\right)^{2}+\left(y_{T_{i}}-y\right)^{2}}+\sqrt{\left(x_{R_{j}}-x\right)^{2}+\left(y_{R_{j}}-y\right)^{2}}}{c_{S 0}}\right)\right|
$$

Where:

- $I_{(x, y)}$ is the intensity of the pixel located at the Cartesian coordinates $(x, y)$

- $\quad h_{T_{i}, R_{j}}$ is the envelope of the signal obtained from the transmitting transducer $T_{i}$ and the receiving transducer $R_{i}$ 
- $\quad x_{T_{i}}$ and $y_{T_{i}}$ are the Cartesian coordinates of the transmitting PZT $T_{i}$

- $\quad x_{R_{j}}$ and $y_{R_{j}}$ are the Cartesian coordinates of sensing (or receiving) PZT $R_{i}$

- $\quad N$ is the total number of PZT transducers

$-\quad c_{S 0}$ is the group velocity

The algorithm was developed and applied to the experimental data using MATLAB. A flow chart of the program is shown in Fig. 14 and summarises the working principle of the TFM with FMC.

Regarding the selection of the resolution, ultimately from a processing point of view the maximum resolution that can be achieved is limited by the sampling frequency of the signal and the distance between sensors. For a given signal, each point obtained after the arrival of the first wave packet (wave travelling directly from the transmitter to the receiver) can be used to calculate a pixel intensity. This means that in the case of a signal containing 100,000 points (such as used in this study) a megapixel resolution could be achieved. However, this does not

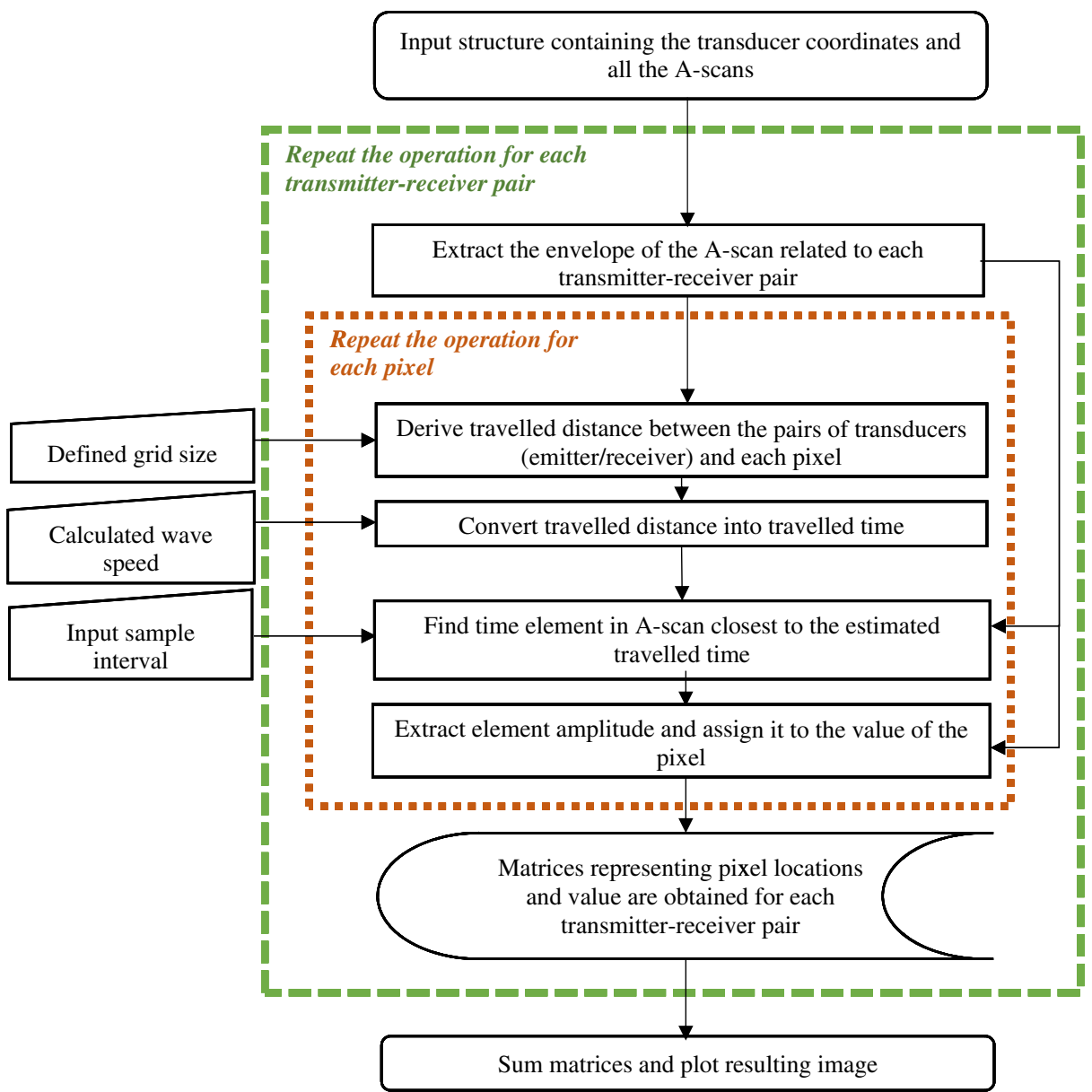

Fig. 14 Flow chart representing the working principle of the program of the TFM with FMC designed in MATLAB 
necessarily mean that the accuracy of the resulting image will be increased as the information provided by the signal also carries inaccuracy relative to the instrumentation and the sensors used. In addition, there is a trade-off between the resolution and computational time: a higher resolution would necessitate a higher computational time.

One issue arising from the implementation of TFM is that every signal showing a defect location (based on reflection) in a pixel is accompanied by at least one, and up to three, artefacts, as shown in Fig. 15. They are generated by the existence of three axes of symmetry passing by the centre of the distance between the transmitting and receiving transducers. The variation in number of artefacts present in the image for a given signal depends on the artefact's location, as only the artefact existing within the boundary will be seen.

\subsection{Resulting Images}

In order to reduce the computing time, the signals were down-sampled to 1,000 points from the initial 100,000 points, prior to being processed with the imaging algorithm. The resulting, normalised images from the application of the TFM to the baseline and Test 1 signal are shown Fig. 16. While the resulting, normalised images from the data obtained after subtraction of the baseline signal from the Test 1 signals is shown in Figs. 17, 18 and 19. The colour chart defining the pixel intensity, present in both figures, is related to the normalised summation of the amplitude extracted from each signal. Note that all images presented in this section contain 100x100 pixels.

The image obtained from the baseline data in Fig. 16a enables the observation of the location of the artefacts inherent to the system, and unrelated to damage. The high intensity (approximately 0.6) of pixels found in the region located within the circular array of sensors is likely to originate from two contributing factors. The first potential enhancing factor can be attributed to the high amplitude found in the A-scan around the arrival time of S0-mode direct path. The second possible factor is the amplitude of the A0-mode, as this mode was highly attenuated, but not completely cancelled at the excitation frequency of $300 \mathrm{kHz}$. A comparison with a simulated signal, which is a potential route for further work, could confirm this theory as well as distinguish the impact each factor has. Other artefacts were found in the region outside the circular array of sensors (Fig. 16a), they form a ring of approximately $1 \mathrm{~m}$ in
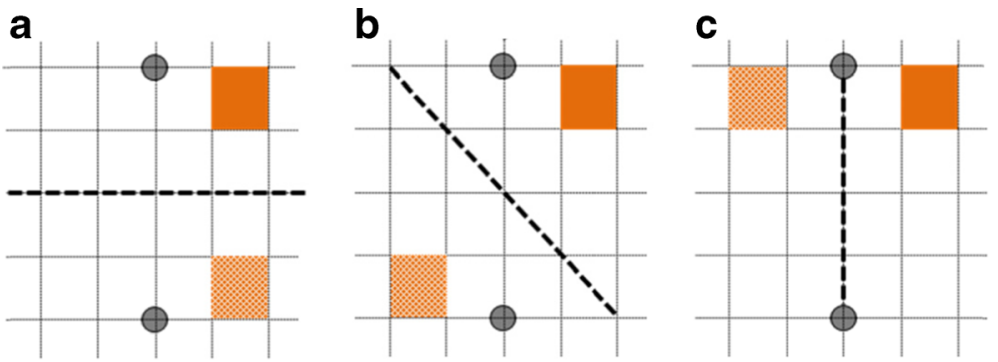

Legend:

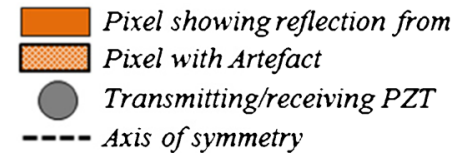

Fig. 15 Schematic of the three possible artefact locations with respect to (a) the horizontal, (b) the diagonal and (c) the vertical axis of symmetry 

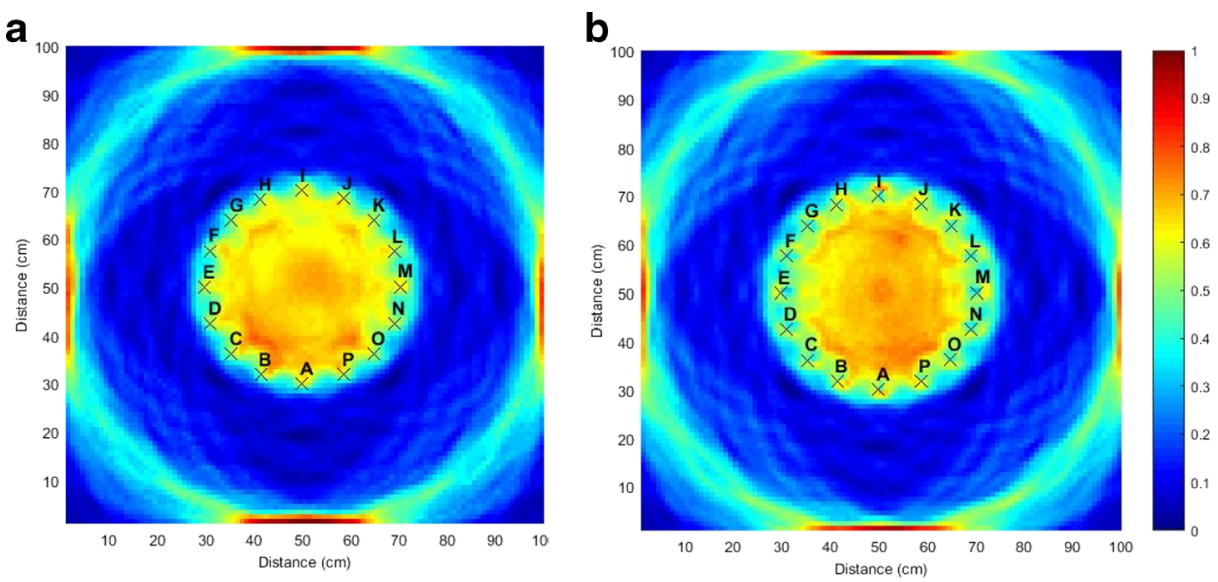

Fig. 16 Image obtained from baseline signal (a) and signal from Test 1 (b) (signal down-sampled to 1000 points)

diameter, crossing the four plate boundaries. These artefacts are consistent with the overlapping of multiple ellipses observed in Fig. 10a. In particular, the pixel intensity is found to be higher along the boundaries where common tangents between the ellipses were found. Therefore, these artefacts can be attributed to the effect of the boundary reflections of S0 mode along the edges of the plate. Similar artefacts are observed on the image in Fig. 16b resulting from Test 1 data (with circular damage). However, by comparing both images in Fig. 16, it can be observed that the pixel intensity in Fig. 16b in the area of the damage location ( $54 \mathrm{~cm}$ on the $\mathrm{x}$-axis and $60 \mathrm{~cm}$ on the y-axis) is higher than on the baseline image (Fig. 16a). Nevertheless, without the baseline image it is difficult to differentiate the pixels representing the damage from the artefacts.

The analysis of the image obtained from the subtracted baseline Fig. 17 shows that a group of pixels with medium intensity (approx. 0.4) circles the damaged area. These pixels relates to the summation of amplitude originating from the reflections of guided waves at the boundary of the damage. Considering that the damage is a hole, these results correlate with the

a

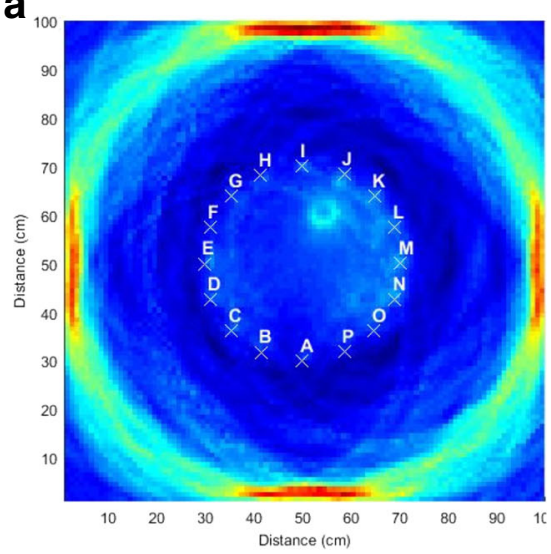

b

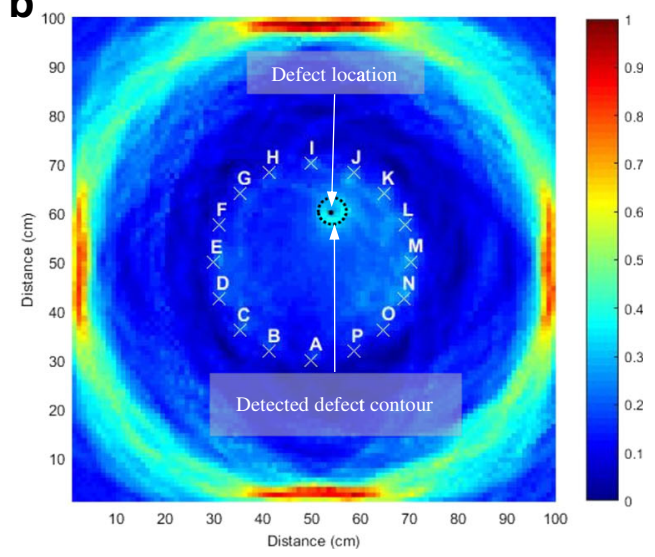

Fig. 17 Image obtained for data from the subtracted baseline to the signals form test 1 with (a) and without (b) showing the actual damage location (signal down-sampled to 1000 points) 


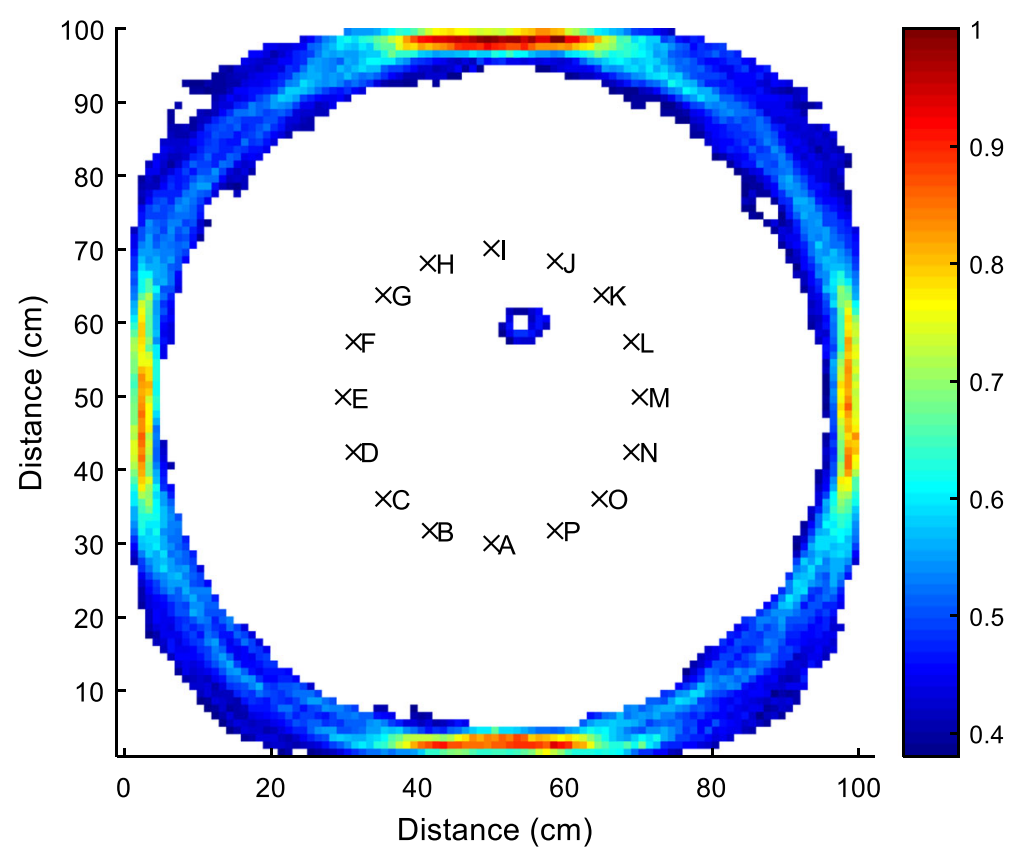

Fig. 18 Image obtained when implementing a filter removing the intensities lower than 0.38 on the image shown in Fig. 17

expectation that high intensity can be observed around the defect boundary, hence highlighting its contour, while inside the damage (where the waves cannot propagate) a lower intensity caused by processing artefacts prevail. A filter removing any normalised intensity lower than 0.38 (based on the intensity range in Fig. 17) was implemented in Fig. 18 in order to remove some of the processing artefacts.

a

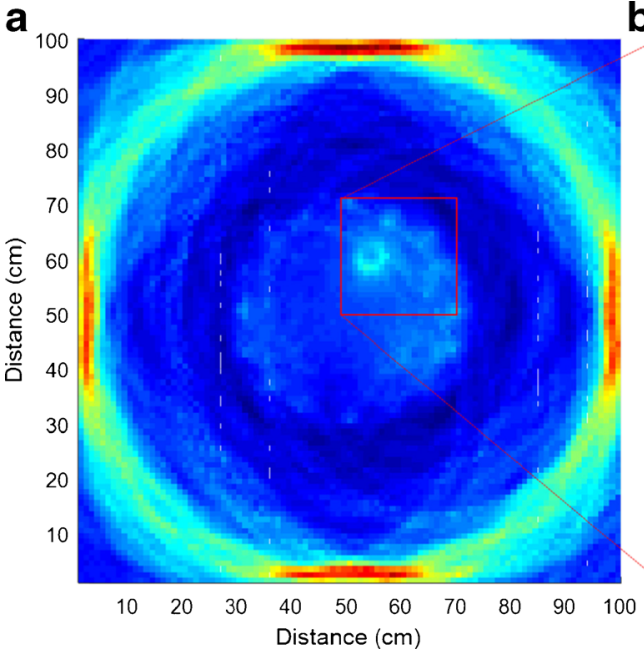

b

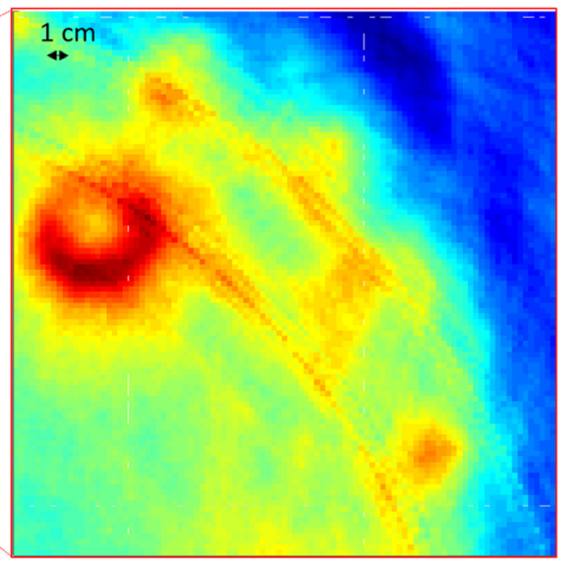

Fig. 19 The resolution was locally increased providing a zoom of $100 \times 100$ pixels around the damage $(\mathbf{b})$, the zoom covered an area of $250 \times 250 \mathrm{~mm}$ its relative position in the plate is shown in (a) 
Although the $10 \mathrm{~mm}$ diameter damage is smaller than the $17 \mathrm{~mm}$ wavelength of the propagating S0 mode, Fig. 17 confirms that guided waves combined with TFM imaging system enables its detection. However, it can be observed on Fig. 17 that the current resolution (1 pixel per centimetres squared) shows blurred damage contour. This was improved by increasing the resolution locally around the damage location, such as shown in Fig. 19. In the zoomed image (Fig. 19b) the resolution remains $100 \times 100$ pixels and covers an area of $25 \times 25 \mathrm{~cm}$. The pixels highlighting the damage contour form a ring (approximately $70 \mathrm{~mm}$ outer diameter and $25 \mathrm{~mm}$ inner diameter) centred on the known damage location. The inner diameter of the ring best described the position of the damage boundary. The accuracy of the damage localisation and sizing is of the order of one millimetre, although further experiment and processing will be necessary to quantify the system accuracy and its repeatability with sufficient certainty. In addition, further study will be required to identify the system's ability to differentiate between neighbouring defects. Based on the current result it can be estimated that smaller damage, probably up to a third of the wavelength, could be identified with this technique.

\section{Conclusions and Suggestion for Further Work}

In conclusion, this study proposed an imaging method for structural health monitoring using guided wave signals post-processed through TFM and FMC algorithms. For each structural assessment, 240 signals were collected experimentally in an isotropic plate using 16 PZT transducers in pitch and catch configuration. All Lamb wave signals were tuned on the fundamental symmetric mode at $300 \mathrm{kHz}$. The wave speed and transducer positions, which are the initial conditions of the imaging system, were determined using a technique based on the processing of the baseline signal and the characterisation of the reflected wave paths. This method prevents the accumulation of errors related to the location of transducers though manual measurement. The imaging system was designed to enable random transducer positions and multiple transmitting and receiving transducers. Data were collected after a circular damage of $10 \mathrm{~mm}$ in diameter was drilled in the plate. The imaging system was then applied to the baseline data, the data of the damage signals and the data obtained from the subtraction of the baseline signals from the damaged signals.

The image of the undamaged plate (with the baseline data) enabled the observation of the location of the different signal processing artefacts unrelated to damage, but inherent to the system. Similar observations were made of the image obtained from the signals collected in the presence of the circular damage, demonstrating the difficulty of identifying inherent defects. A filtered image has shown the possibility of removing some of those processing artefacts. The last images obtained from the subtracted data enabled the identification of the contour of the damage, hence enabling an appreciation of the defect location and size. The defect was observed within a millimetre distance from its actual position, and based on the contour its maximum size was expected to be less than $25 \mathrm{~mm}$. Still, these results are in good agreement with the actual damage. The main achievement of the imaging system was its ability to detect and locate a defect with a diameter $40 \%$ smaller than the $17 \mathrm{~mm}$ wavelength of the fundamental symmetric mode. According to these results it can be estimated that smaller damage, up to one third of the wavelength of S0 mode, could still be identified.

Future investigations will evaluate the ability of the system to differentiate neighbouring defects, by quantifying the minimum distance required to do so, and the degree of confidence. 
Additionally, the system will be tested in a CFRP plate. The application of the wave reflections and image processing will require accounting for the damping related to fibre orientation which will also affect the speed of the guided waves.

Acknowledgments This research was supported by the Aerospace Research Institute of the University of Manchester.

Open Access This article is distributed under the terms of the Creative Commons Attribution 4.0 International License (http:/creativecommons.org/licenses/by/4.0/), which permits unrestricted use, distribution, and reproduction in any medium, provided you give appropriate credit to the original author(s) and the source, provide a link to the Creative Commons license, and indicate if changes were made.

\section{References}

1. Giurgiutiu, V.: Structural Health Monitoring with Piezoelectric Wafer Active Sensors. Elsevier Inc., IBSN13: 978-0-12-088760-6 (2008)

2. $\mathrm{Su}, \mathrm{Z}$., and Ye, L.: Identification of Damage Using Lamb waves From Fundamentals to Applications. F. Pfeiffer, P. Wriggers (Eds), vol. 48, ISBN: 978-1-84882-783-7 (2009)

3. Gresil, M., and Giurgiutiu, V.: Prediction of attenuated guided waves propagation in carbon fiber composites using Rayleigh damping model. Journal of Intelligent Materials Systems and Structures, 26(16) (2015)

4. Ihn, J.B., Chang, F.K.: Detection and monitoring of hidden fatigue crack growth using a built-in piezoelectric sensor/actuator network: I. Diagnostics. Smart. Mater. Struct. 13, 609-620 (2004)

5. Diamanti, K., Soutis, C.: Structural health monitoring techniques for aircraft composite structures. Prog. Aerosp. Sci. 46(8), 342-352 (2010)

6. Grondel, S., Assaad, J., Delebarre, C., Moulin, E.: Health monitoring of a composite wingbox structure. Ultrasonics 42(1-9), 814-824 (2004)

7. Willberg, C., Koch, S., Mook, G., Pohl, J., and Gabbert, U.: Continuous mode conversion of Lamb waves in CFRP plates. Smart Materials and Structures, 21 (2012)

8. Staszweski, W.J., Mahzan, S., Traynor, R.: Health monitoring of aerospace composite structures - Active and passive approach. Compos. Sci. Technol. 69(11-12), 1678-1685 (2008)

9. Santhanam, S., Demirli, R.: Reflection of Lamb waves obliquely incident on the free edge of a plate. Ultrasonics 53, 271-282 (2012)

10. Giridhara, G., Rathod, V.T., Naik, S., Roy Mahapatra, D., Gopalakrishnan, S.: Rapid localization of damage using circular sensor array and Lamb wave based triangulation. Mech. Syst. Signal Process. 24, 2929-2946 (2010)

11. Yu, L., and Giurgiutiu, V.: Improvement of Damage Detection with the Embedded Ultrasonics Structural Radar for Structural Heath Monitoring. Proceedings of 5th International Workshop on Structural Health Monitoring, Stanford University, Stanford, USA (2005)

12. Fan, C., Caleap, M., Pan, M., Drinkwater, B.W.: A comparison between ultrasonic array beamforming and super resolution imaging algorithms for non-destructive evaluation. Ultrasonics 54, 1842-1850 (2014)

13. Hall, J.S., Michael, J.E.: Multipath ultrasonic guided wave imaging in complex structures. Struct. Health Monit. 14(4), 345-358 (2015)

14. Stepinski, T., Ambrozinski, L., and Uhl, T.: Beamforming of Lamb waves using 2D arrays: A comparative study. Structural Health Monitoring 2013, ed. Fu-Kuo Chang, Proceedings of the 9th International Workshop on SHM Stanford University, Stanford, USA, 2210-2217 (2013)

15. Lissenden, C. J., and Rose, J. L.: Structural Health Monitoring of Composite laminates Through Ultrasonic Guided Wave Beam Forming. Proceedings of NATO Applied Vehicle Technology Symposium on Military Platform, RTO-MP-AVT-157 (2014)

16. Knight-Gregson, B., Mosey, S., Weston, M., Scully, P., Anthony Peyton, A., and Davis, C.: Advanced photo-acoustic imaging using full matrix capture data acquisition. 4th International Symposium on Laser Ultrasonics \& Advanced Sensing, Evanston, USA (2015)

17. Schmitz, V., Chakhlov, S., Müller, W.: Experiences with synthetic aperture focusing technique in the field. Ultrasonics 38(1), 731-738 (2000). doi:10.1016/S0041-624X(99)00219-X

18. Spies, M., Jager, W.: aperture focusing for defect reconstruction in anisotropic media. Ultrasonics 41(2), 125-131 (2003). doi:10.1016/S0041-624X(02)00407-9

19. Thomson, R.N.: Transverse and longitudinal resolution of the synthetic aperture focusing technique. Ultrasonics 22(1), 9-15 (1984) 
20. Doctor, S.R., Hall, T.E., Reid, L.D.: SAFT - the evolution of a signal processing technology for ultrasonic testing. NDT Int. 19(3), 163-167 (1986). doi:10.1016/0308-9126(86)90105-7

21. Masri, W. M.: Diffraction-corrected synthetic aperture focusing for spherical ultrasonic radiators. Retrospective Theses and Dissertations, Iowa State University, USA (1997)

22. Kramer, S.: Ultrasonic weld defect sizing using the synthetic aperture focusing technique. Rev. Prog. Quant. Nondestruct. Eval. 8(9), 1995-2002 (1989). IBSN: 978-1-4612-8097-2

23. Gaydecki, P.A., Burdekin, F.M., Damaj, W., John, D.G., Payne, P.A.: Digital deconvolution analysis of ultrasonic influenced by the presence of longitudinally aligned steel cables in pre-stressed concrete. Meas. Sci. Technol. 3(9), 909-917 (1992)

24. Schikert, M., Krause, M., Muller, W.: Ultrasonic imaging of concrete elements using reconstruction by synthetic aperture focusing technique. J. Mater. Civ. Eng. 5(3), 235-246 (2003)

25. Shandiz, H.T., Gaydecki, P.A.: An enhanced, high-speed ultrasonic SAFT system for imaging of defects within concrete structures. Am. Inst. Phys. Conf. Proc. 557, 703 (2001)

26. Wiggenhauser, H., Niederleithinger, E.: Innovative ultrasonic techniques for inspection and monitoring of large concrete structures. EPJ Web Conf. 56, 04004 (2013). doi:10.1051/epjconf/20135604004

27. Gaul, T., Schubert, L., Weihnacht, B., and Frankenstein, B.: Localization of defects in pipes using guided waves and synthetic aperture focussing technique (SAFT). Proceedings of the 7th European Workshop on Structural Health Monitoring, Nantes, France (2014)

28. Li, F., Peng, H., Meng, G.: Quantitative damage image construction in plate structures using a circular PZT array and Lamb waves. Sensors Actuators A 214, 66-73 (2014)

29. Shen, Y., Giurgiutiu, V.: WaveFormRevealer: an analytical framework and predictive tool for the simulation of multi-modal guided wave propagation and interaction with damage. Struct. Health Monit. - Int. J. 13(5), 491-511 (2014). doi:10.1177/1475921714532986

30. Gresil, M., Giurgiutiu, V.: Prediction of attenuated guided waves propagation in carbon fiber composites using Rayleigh damping model. J. Int. Mater. Syst. Struct. (2014). doi:10.1177/1045389X14549870 